\title{
The impact of programs for prevention of mother-to-child transmission of HIV on health care services and systems in sub-Saharan Africa - A review
}

\author{
Jean Claude Mutabazi ${ }^{1,2^{*}}$ (D), Christina Zarowsky ${ }^{1,2,4}$ and Helen Trottier ${ }^{1,3}$
}

\author{
* Correspondence: \\ mutajeanc@yahoo.fr \\ 'Département de Médecine Sociale \\ et Préventive, École de Santé \\ Publique, Université de Montréal, \\ Pavillon 7101, Avenue du Parc, \\ Montreal, QC H3N 1X7, Canada \\ ${ }^{2}$ Centre de Recherche du Centre \\ Hospitalier de l'Université de \\ Montréal (CRCHUM), Tour \\ Saint-Antoine, 3rd Floor, Room: \\ S03.516, 900, Rue St-Denis, \\ Montreal, QC H2X 0A9, Canada \\ Full list of author information is \\ available at the end of the article
}

\begin{abstract}
Background: The global scale-up of Prevention of mother-to-child transmission (PMTCT) services is credited for a 52\% worldwide decline in new HIV infections among children between 2001 and 2012. However, the epidemic continues to challenge maternal and paediatric HIV control efforts in Sub Saharan Africa (SSA), with repercussions on other health services beyond those directly addressing HIV and AIDS. This systematised narrative review describes the effects of PMTCT programs on other health care services and the implications for improving health systems in SSA as reported in the existing articles and scientific literature. The following objectives framed our review:

1. To describe the effects of PMTCT on health care services and systems in SSA and assess whether the PMTCT has strengthened or weakened health systems in SSA

2. To describe the integration of PMTCT and its extent within broader programs and health systems.
\end{abstract}

Methods: Articles published in English and French over the period 1st January 2007 (the year of publication of WHO/UNICEF guidelines on global scale-up of the PMTCT) to 31 November 2016 on PMTCT programs in SSA were sought through searches of electronic databases (Medline and Google Scholar). Articles describing the impact (positive and negative effects) of PMTCT on other health care services and those describing its integration in health systems in SSA were eligible for inclusion. We assessed 6223 potential papers, reviewed 225, and included 57.

Results: The majority of selected articles offered arguments for increased health services utilisation, notably of ante-natal care, and some evidence of beneficial synergies between PMTCT programs and other health services especially maternal health care, STI prevention and early childhood immunisation. Positive and negative impact of PMTCT on other health care services and health systems are suggested in thirty-two studies while twenty-five papers recommend more integration and synergies. However, the empirical evidence of impact of PMTCT integration on broader health systems is scarce. Underlying health system challenges such as weak physical and human resource infrastructure and poor working conditions, as well as social and economic barriers to accessing health services, affect both PMTCT and the health services with which PMTCT interacts.

(Continued on next page)

\section{Biomed Central}

(c) The Author(s). 2017 Open Access This article is distributed under the terms of the Creative Commons Attribution 4.0 International License (http://creativecommons.org/licenses/by/4.0/), which permits unrestricted use, distribution, and reproduction in any medium, provided you give appropriate credit to the original author(s) and the source, provide a link to the Creative Commons license, and indicate if changes were made. The Creative Commons Public Domain Dedication waiver (http://creativecommons.org/ publicdomain/zero/1.0/) applies to the data made available in this article, unless otherwise stated. 
(Continued from previous page)

Conclusions: PMTCT services increase to some extent the availability, accessibility and utilisation of antenatal care and services beyond HIV care. Vertical PMTCT programs work, when well-funded and well-managed, despite poorly functioning health systems. The beneficial synergies between PMTCT and other services are widely suggested, but there is a lack of large-scale evidence of this.

Keywords: Maternal and child health, PMTCT, Health systems integration, Sub Saharan Africa

\section{Background}

The global scale-up of Prevention of Mother-To-Child Transmission (PMTCT) services is credited for a $52 \%$ worldwide decline in new Human Immunodeficiency Virus (HIV) infections among children between 2001 and 2012 [1]. With adequate efforts, more funding and closely monitored progress, the United Nations Programme on HIV and AIDS (UNAIDS) still reported 160,000 new paediatric HIV infections in 2016 [2]. Despite significant progress, the epidemic continues to challenge maternal and paediatric services in Sub Saharan Africa (SSA), with repercussions on other health services beyond those directly addressing HIV and Acquired Immunodeficiency Syndrome (AIDS) [3].

This systematised narrative review describes the impact and integration of PMTCT programs on other health care services. We begin with an overview of prevention of mother-to-child transmission of HIV in the context of global AIDS control efforts, as well as an introduction to the concepts of integration of "vertical" or disease-based programs into broader health systems. We then present and discuss the findings of our review after having presented the methodology.

\section{Overview: HIV, PMTCT, and health system integration}

Preventing - and eliminating - mother-to-child transmission of HIV

The highest prevalence of HIV infection is in sub-Saharan Africa, where rates of both prevalent and new infections are consistently higher among women than men and most women are now diagnosed during pregnancy or at delivery through antenatal and perinatal care [4]. The Mother-To-Child Transmission (MTCT) of HIV refers to the spread of HIV from an HIV positive woman to her child either during pregnancy, childbirth (labour, delivery) or breastfeeding. MTCT is the most common mode of transmission of HIV to children. Over $90 \%$ of children are infected with HIV through MTCT [5]. The interventions aiming to prevent paediatric HIV/AIDS and for better health of both mothers and their children are known as "prevention of mother to child transmission of HIV" or PMTCT [6].

Since MTCT can be prevented through antiretroviral therapy (ART), a global campaign for its virtual elimination was launched on 21 May 2009 as one of the top priorities for the World Health Organisation (WHO), the Joint United Nations Programme on HIV and AIDS (UNAIDS), the United Nations Children's Fund (UNICEF) and the United Nations Population Fund (UNFPA) [7]. The policy of eliminating new paediatric HIV infections depends on countries reaching not only high rates of initiating ART among pregnant HIV-infected women but also maintaining and supporting them to adhere to treatment [8]. 


\section{PMTCT interventions}

PMTCT, also called "prevention of vertical transmission", [9] has been widely implemented in hospital and clinic services particularly in those dealing with antenatal, perinatal and postpartum care. In 2007, WHO and UNICEF developed a guideline document to scale up PMTCT, focusing on resource-constrained settings and efforts towards universal access for women, infants and young children in order to eliminate HIV and AIDS among children [10]. The intervention elements to prevent MTCT are known as the PMTCT cascade [6,10], outlined in summary form (Table 1).

This PMTCT cascade reduces the chances for HIV to pass from an HIV-positive mother to her baby during pregnancy, labour, or delivery, or through breastfeeding $[5,6,11]$. Four components of the comprehensive PMTCT programme are articulated by WHO and UNICEF, namely: (1) primary prevention of HIV infection among women of childbearing age; (2) preventing unintended pregnancies among women living with HIV;

(3) preventing HIV transmission from a woman living with HIV to her infant; and (4) providing appropriate treatment, care and support to mothers living with HIV and their children and families $[6,10]$. The PMTCT cascade is partly or fully implemented by many actors from public and private for-profit and not-for-profit health care sectors (Non-Governmental Organisations (NGOs)), religious and community groups) operating locally but managed and funded at local, national and international levels [12-14].

These multiple actors with their diverse agendas and policies initially delivered PMTCT services as a stand-alone and externally funded programme. The programmes progressively won the interest of governments and are now increasingly supported through public funding in many countries, while still requiring substantial donor support [15]. With time, the strong links this cascade has with maternal and child health services required closer collaboration with and increasing integration into broader services towards sustainable outcomes $[14,16]$.

Table 1 PMTCT cascade

PMTCT CASCADE (focusing primarily on components 3 and 4, preventing transmission to infant and treating mother and infant)

\begin{tabular}{lll}
\hline Component & Linked to & All pregnant \\
\hline 1. Utilisation of antenatal care (ANC) & ANC services & women \\
2. Receiving HIV pretest counselling & ANC unit or voluntary counselling & and testing (VCT) services \\
3. Acceptance of HIV test & ANCNCT services & HIV- positive \\
4. Receiving HIV test results \& post-test & ANCNCT services \\
counselling & Pregnant women \\
5. Get CD4 assessment & ANCNCT services \\
$\begin{array}{l}\text { 6. Use of ARV prophylaxis for mom and/or } \\
\text { baby (for seropositive moms) }\end{array}$ & \\
7. Adherence to ARVs during pregnancy & ANC unit/ARV programs \\
8. Deliver with skilled attendant \& Take ARVs & Obstetric services \\
9. Follow safe infant feeding practices & Child health services \\
10. Bring infant for HIV testing & Child health or VCT services \\
11. Adhere to maternal/infant ARVs after birth & Child health, obstetric or VCT services \\
12. Use postpartum family planning & Obstetric services \\
\hline
\end{tabular}


The worldwide expanded access to PMTCT services prevented more than 670,000 children from acquiring HIV from 2009 to 2012 [17]. In 2012, over 900,000 pregnant women living with HIV globally accessed PMTCT services - a coverage of $62 \%$ - and only 160,000 new paediatric HIV infections were reported in 2016, compared to 300,000 in 2010 [2]. Four African countries (Botswana, Ghana, Namibia and Zambia) had achieved 90\% PMTCT coverage [17] while the PMTCT coverage was over 90\% and over $80 \%$ in Rwanda and South Africa respectively by 2014 [18, 19].

Based on these promising PMTCT figures in SSA, it is possible to envisage achieving virtual elimination of MTCT. In addition, one could expect to see effects of PMTCT programs on other health care services because PMTCT is now largely implemented through existing maternal health programs and services. However, effects on health services and systems are likely to be uneven and complex.

PMTCT is a complex intervention with many involved actors and policies, flows of knowledge, materials, technologies and funds, interacting at global, national and local levels $[20,21]$. Beyond the operational challenges of actually delivering the cascade on the ground, this complexity and the history of HIV control programs globally raise systemic and political issues related to the involvement of external funders, experts and manufacturers - sometimes called the global AIDS industry - in funding and implementing HIV services in low and middle-income countries [22]. In addition, PMTCT programs, especially those which aim to be integrated in and help to strengthen health services and systems, seek to address three health goals, each of which is itself a major effort involving different actors, structures and health strategies. These three goals are: combatting HIV/AIDS, reducing child mortality and improving maternal health $[12,23]$. Since health services in many countries are organised, funded and managed to deal with distinct diseases and populations but are seen as parts of an overall health system, these multiple interfaces raise questions of whether PMTCT programs have an overall weakening or strengthening impact on national health systems, or no impact at all beyond PMTCT services. For example, Rwanda's success in scaling up paediatric HIV services through effective utilisation of health resources may offer lessons for other developing countries with high prevalence of maternal and paediatric HIV. This successful integration of PMTCT in Rwanda may be attributed to its health system organisation, despite the weakness of the system [24]. Even so, in contrast to these reported good PMTCT outcomes in countries with a history of stagnating health systems like Rwanda (and most other SSA countries), there is little evidence-informed meaningful discussion about possible PMTCT effects on other maternal and child health services and on overall health systems. This review examines some of these questions.

\section{PMTCT integration}

If we conceptualise PMTCT as a service within a "continuum of care" approach addressing different periods of women's and children's life course, PMTCT programs could be seen to have potential beneficial synergies with other reproductive, maternal and child health interventions such as Sexually Transmitted Infection (STI) control, early childhood immunisation, antenatal and delivery care, family planning, nutrition supplementation [16]. For example, maternal deaths have been declining at $2.3 \%$ 
worldwide but this reduction is at only $1.7 \%$ in SSA where $9 \%$ of all maternal deaths were estimated to be due to HIV/AIDS. The slower rate of decline in maternal deaths in SSA has stimulated interest in a range of recent strategies to increase the coverage of good health care, notably free or very low-cost obstetrical care [25]. The improvement in HIV-related care of pregnant women should help to increase the impact of these other strategies, and thus contribute to accelerated decreases in maternal mortality in high-HIV burden countries.

The potential impact of PMTCT on health services may go beyond maternal and child health specialities and may involve other health care services indirectly, with potential opportunities to enhance overall quality of care but also posing threats such as brain drain and redirection of resources from programs not related to HIV in contexts where there is already weak infrastructure. This raises questions regarding the appropriate approaches to address the challenges regarding the accessibility, equity, and quality of healthcare in the efforts to facilitate service delivery and strengthen health systems [26].

The global monitoring framework and strategy put in place towards elimination of new HIV infections in children [12], reflects this wider perspective of integration across programs and services and calls for comprehensiveness through seven priority areas. These priorities are: (i) Ensure leadership and country ownership; (ii) Improve coverage, access and utilisation of services; (iii) Strengthen quality of Maternal, New born and Child Health services to deliver effective PMTCT of HIV and syphilis interventions; (iv) Enhance provision of linked services; (v) Strengthen human resource capacity, supply chain management and information systems; (vi) Improve measurement of performance and impact and (vi) Develop and engage community systems [12]. This framework does not take PMTCT as a self-sufficient entity but instead calls for seeing PMTCT as integrated within a health system. It thus echoes the WHO-recommended integration of PMTCT programs with other healthcare services to achieve the more accessibility and improvement of health interventions [27]. But what does integration entail, analytically and in practice?

\section{Health system integration: Vertical, horizontal and diagonal approaches}

The 2003 work of Oliveira-Cruz et al. on the synergies between vertical and horizontal health interventions [28], remains relevant today. They define vertical programs as free-standing programs delivering health care services, put in place to deal with a particular disease or condition with predetermined goals and designed for a known time frame and calculated financial means. Horizontal programs refer to service delivery through the infrastructure of the regular healthcare system [28]. While exploring the impact of vertical programs on health systems and experiences of integrating these programs, these authors encouraged the concerted use of both modes of delivery, according to the capacity of a health system as it changes over time [29]. The combination of both delivery models suggests what Julio Frenk and others refer to as a "diagonal approach" [15]. The "diagonal approach" has been referred to as using a disease-specific intervention (e.g. HIV/PMTCT) to strengthen a general health system [15]. Working together across initiatives can bring more benefits; for example, the major investments in vertical programs addressing 
specific diseases or conditions such as HIV can through diagonal financing cross-subsidise other programs and also the overall functioning of the health system because "horizontal" services like labs and human resources are essential to implement the AIDS, TB and malaria programs [15].

There is no internationally recognised definition of integrated care and to further complicate analysis, some researchers who address what could be considered as integrated service delivery do not use "integration" as a keyword [27]. Integrated care describes a range of organisational arrangements with variable nature and intensity and comprises two main concepts: a) an organisational structure focused on economic benefits, notably efficiency gains, or b) a way of organising service delivery: from no integration, to partial integration or full integration [30, 31]. For both of these approaches, integration is a process that "occurs at different levels of the health system (regional, district, health facility) and in relation to key health system functions namely governance, financing, planning, service delivery, monitoring and evaluation, demand generation" [27, 32]. Analysis of integrated health care requires a good understanding of health care services and health systems components and functions, and integration must be viewed as a process that accommodates complex agendas and issues as was concluded in a study of postnatal care integration into PMTCT in Swaziland [31, 33].

In terms of implementation practices, PMTCT interventions may be carried out in one or more of the following health services: (1) antenatal clinic, (2) delivery/obstetric/ labour ward care, (3) postnatal care, (4) neonatal/new born care, (5) paediatric/infant care, (6) nutritional programs, (7) HIV testing and support centres, (8) HIV treatment centres, (9) reproductive/gynaecological services, (10) sexually transmitted infection clinics, (11) family planning, (12) primary health care which in many settings may be largely focused on conditions such as malaria, acute respiratory infection, diarrhoea and malnutrition, (13) Emergency care, (14) Tuberculosis clinics, (15) Malaria clinics (in areas with high burdens of malaria and HIV), (16) Immunisation, (17) or other service [27]. It is worth underlining the close ties between HIV including PMTCT and tuberculosis or/and malaria in terms of coinfection, prevalence, targeted groups and global funding initiatives [34-36]. This variety of potential entry points in the context of the discussion of potential synergies suggests how, and how much, the integration or non-integration of PMTCT could contribute to overall strengthening and integration or weakening and fragmentation of services and systems. In any case, the outcomes depend on how actors collaborate between themselves at health system level, and with the community or individuals seeking health care. A constructive cooperation is encouraged [30,37] to make integration work and minimise its potential negative effects.

One example that demonstrates the need for cooperative efforts to avoid negative effects of a vertical program on health systems and communities relates to the universal child immunisation goals. A study in six countries in Africa and Asia documented how a top-down model in immunisation interventions ended up creating conflicts between local demand and targets of immunisation policy, leading the authors to argue for more intersectoral collaboration if a specific programme is delivered and managed in a vertical way [28]. This example is one among many that supports calls for shifting from a vertical view of PMTCT programs of HIV prevention and treatment towards a horizontal focus on maternal health care and other health care services [14, 38]. 
Unfortunately, the lessons that might be learned from immunisation programs and applied to thinking about PMTCT and health systems have not yet been taken fully into account.

Building on this overview of PMTCT and health system integration, this review sought to describe: (1) the effects of PMTCT on health care services and systems in SSA (2) the integration of PMTCT within broader programs and health systems and extent to which it occurred, and a related question (3) whether health systems as a whole have been strengthened or weakened in countries of SSA.

\section{Methods}

Objectives

This systematically conducted narrative review [39] was based on the following two objectives:

1. To describe the impact of PMTCT on health care services and systems in SSA and assess whether the PMTCT has strengthened or weakened health systems in SSA

\section{To describe the integration of PMTCT and its extent within broader programs and health systems}

\section{Search strategy}

We searched Medline and Google Scholar databases for papers published in English or French between 1st January 2007 (the year of publication of guidelines on global scale-up of the PMTCT of HIV by WHO, UNICEF in partnership with other institutions) and October 2016. We complemented the Medline search with the Google scholar database, looking for any extra articles or grey literature such as policies and programme evaluations important to our review objectives. The following combined search terms were used:

Search 1: (prevention of mother to child transmission or MTCT or PMTCT or (Transmission* or spread*)) adj3 (Mother* adj3 child*)) or HIV or AIDS or acquired immune deficiency syndrome).mp. [ $\mathrm{mp}=$ title, abstract, original title, name of substance word, subject heading word, keyword heading word, protocol supplementary concept word, rare disease supplementary concept word, unique identifier].

Search 2: (Healthcare or Health care or Primary care or Health systems or Health services or community health systems or Primary health care or Maternal health services or Delivery of care or Health facility).mp. [mp = title, abstract, original title, name of substance word, subject heading word, keyword heading word, protocol supplementary concept word, rare disease supplementary concept word, unique identifier].

Search 3: (sub-Saharan Africa or Africa or Africa south of the Sahara or western africa or eastern africa or central africa or southern africa).mp. [mp = title, abstract, original title, name of substance word, subject heading word, keyword heading word, protocol supplementary concept word, rare disease supplementary concept word, unique identifier]. 
The identified papers through these three combined searches were all exported to Endnote bibliographic software [40].

The search strategy did not explicitly require the terms "impact" and "integration" because we felt that some studies might address "impact" and "integration" indirectly, through services description. We defined impact based on the existence of one or more areas of public health action framework as demonstrated through the five aspects of Frieden's 2010 pyramid that underlies intervention with potential health impact [41]. These five aspects are: 1) socioeconomic factors, 2) changing the context to make individual's default decision healthy, 3) long lasting protective interventions, 4) clinical care, 5) counselling and education [41]. Integration was defined based on the concept of integrated care as it was above explained.

\section{Inclusion and exclusion}

We designed the review inclusion and exclusion criteria for the purpose of retaining all studies pertaining to comprehensive PMTCT components throughout health systems in SSA as discussed in the WHO PMTCT strategy [6]. We looked for the papers that examined this strategy in terms of PMTCT's positive and negative effects on other health care services or vice versa, and integration of PMTCT programs with other health care services in the paper's title, results and discussion.

The abstracts of pertinent papers were then retrieved following these inclusion criteria before selecting the full articles: (i) Papers - Research articles published in peer-reviewed scientific journals, grey literature and commentaries dealing with PMTCT in pregnant women in SSA were accepted for inclusion. (ii) Participants - Women at risk of transmitting HIV infection to their children. This could include pregnant women or those at risk of pregnancy and their children, regardless of HIV status. (iii) Interventions All interventions to prevent or reduce HIV MTCT, including but not limited to strategies for antiretroviral therapy and replacement feeding. PMTCT collaboration with other health care services especially maternal and child health $(\mathrm{MCH})$ were included. The following types of articles were excluded: (i) Studies focusing on countries other than SSA countries, (ii) Studies focusing on general HIV/AIDS prevention or other health care services without reference to PMTCT and (iii) Editorials or commentaries generally describing the PMTCT programs on one or more pre-specified healthcare services without studying its effects or integration.

\section{Data extraction and analysis}

All three reviewers agreed on search strategy and inclusion and exclusion criteria. The initial database was created from the compiled electronic searches by one reviewer. All citations were firstly screened by title and abstract and duplicate citations were eliminated. The full texts of potentially eligible papers were then independently obtained for further screening. After resolving differences in data extraction or interpretation through consensual discussions based on the above stated inclusion and exclusion criteria, the final retrieval of papers was conducted. The following study characteristics and data were extracted from included papers: authors, year of publication and country of study, study types or designs, paper's focus interface with PMTCT, brief topic of 
investigation and main results. The findings were organised according to the objectives and referred to as 'themes' pertaining to each of two review objectives.

Throughout the whole selection process, the impact of PMTCT programs on other health care services or vice versa was reported in order to describe to how and to which extent it occurred. Integration of PMTCT programs with other health care services at different levels was also described across all included papers. Two guidelines on global scale-up of the PMTCT were also retrieved.

\section{Results}

We identified 6223 citations and ultimately retained 57 articles, in addition to the PMTCT guidelines mentioned above. These two guidelines are: 1) The global plan for elimination of new HIV infections among children by 2015 and keeping their mothers alive, and 2) The PMTCT strategic vision 2010-2015: preventing mother-to-child transmission of HIV to reach the United Nations General Assembly Special Session (UNGASS) and Millennium Development Goals [6, 42, 43]. The study selection process is presented in the form of an adapted PRISMA flow-diagram (Fig. 1) and the retained articles are summarised in Table 2.

Table 2 describes the included studies in which different types of research designs and data collection methods were used. Two papers were categorised as policy-evaluative (one that evaluates PMTCT policies and one that assesses various national strategic plans in 20 countries in Eastern and Southern Africa). One descriptive study presented a health program. Eighteen reviews, nine qualitative studies, thirteen quantitative studies, and eight mixed methods studies were selected along with six controlled trials and one quasi-experimental study. In most of the studies, hospital and clinical records were reviewed and individual or group interviews with women and staff members involved in health care and PMTCT services were conducted by the researchers

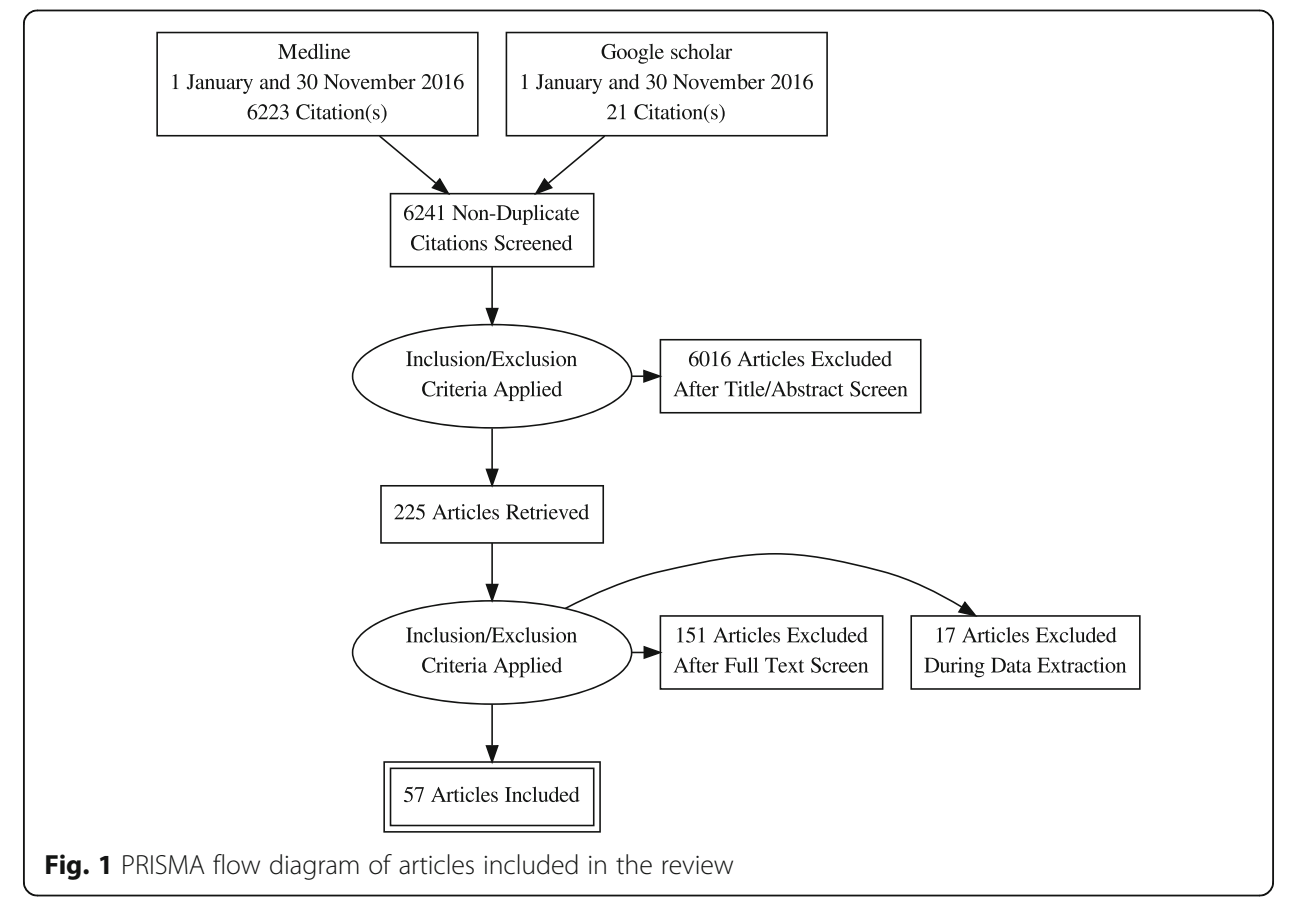




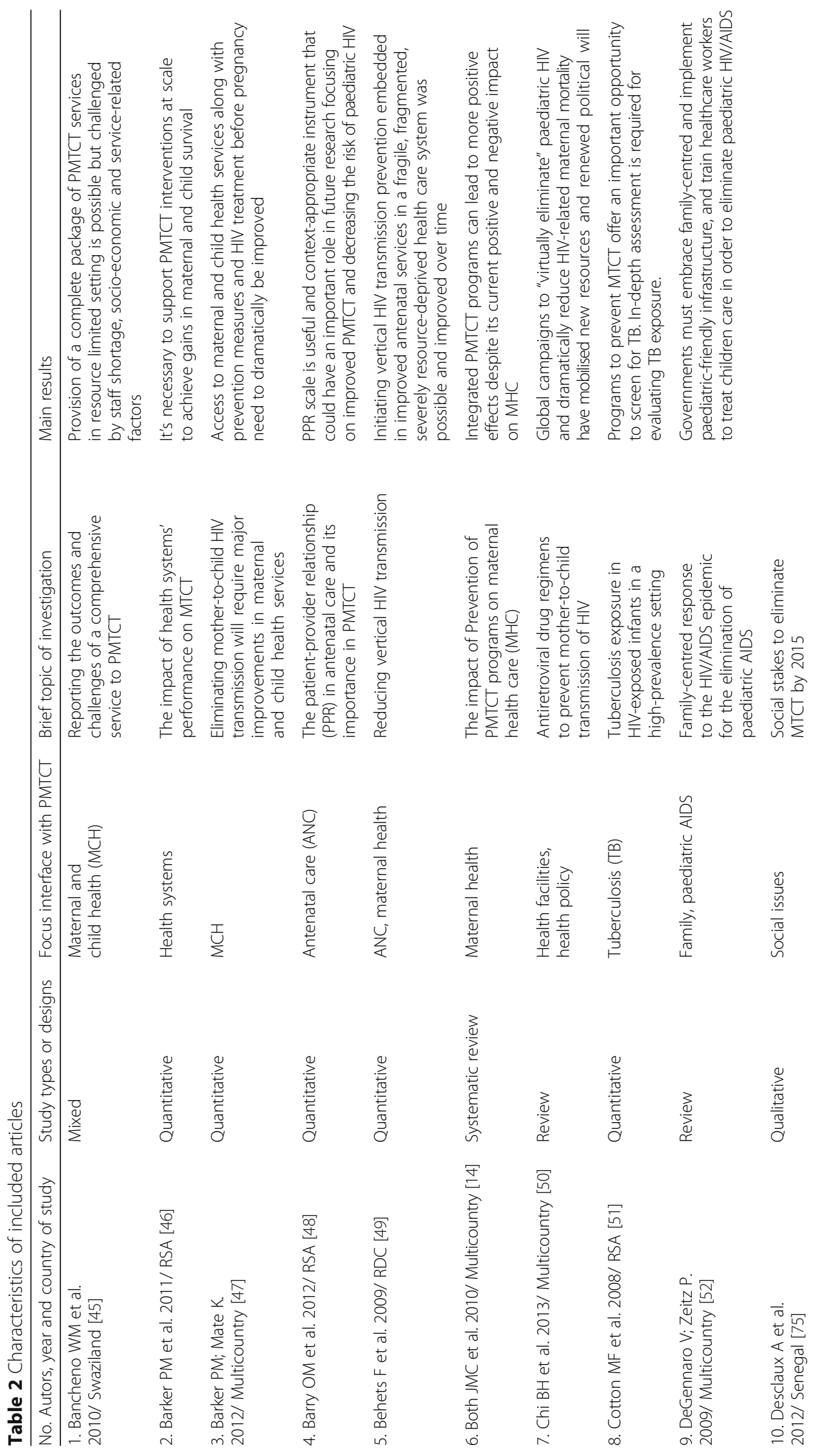




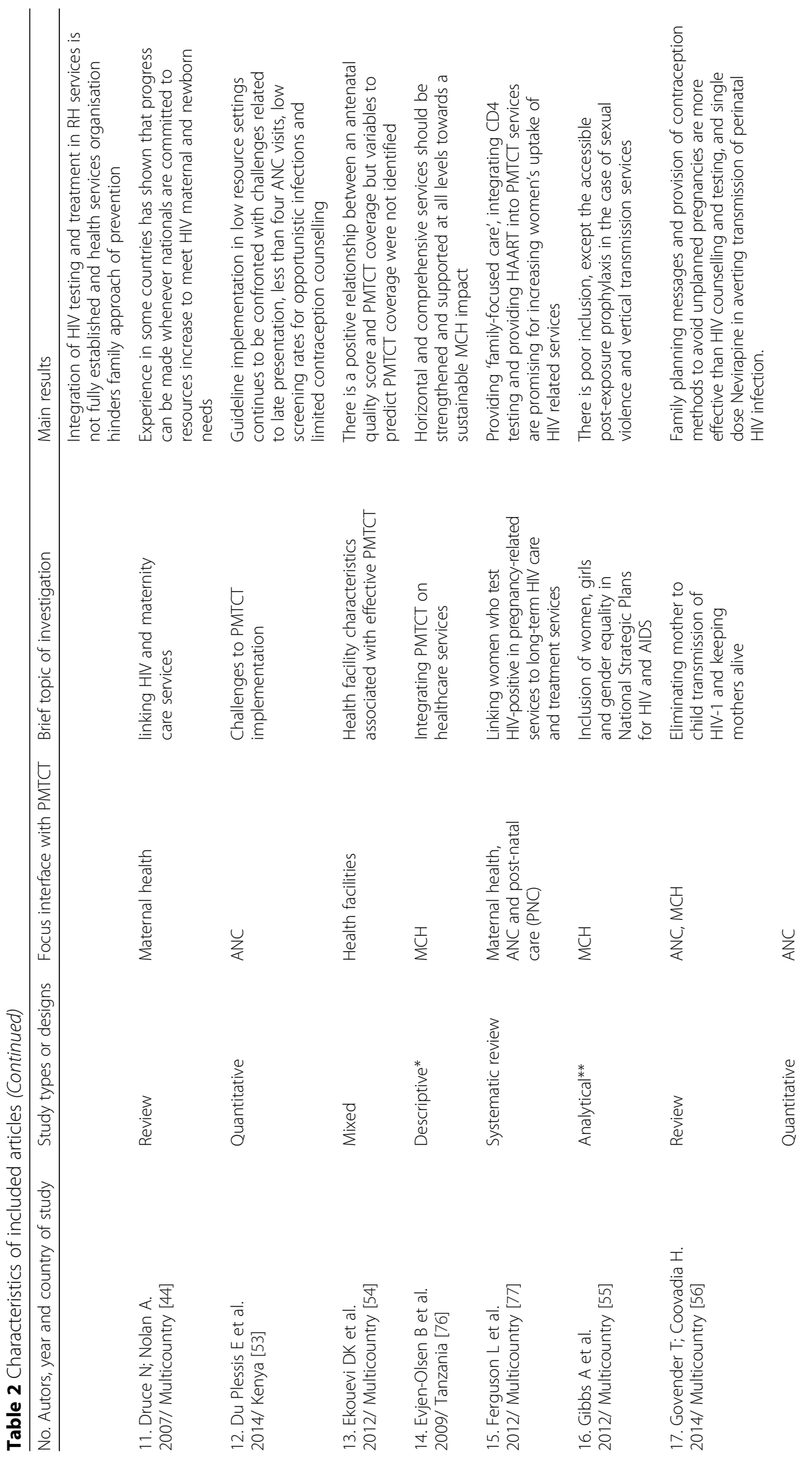




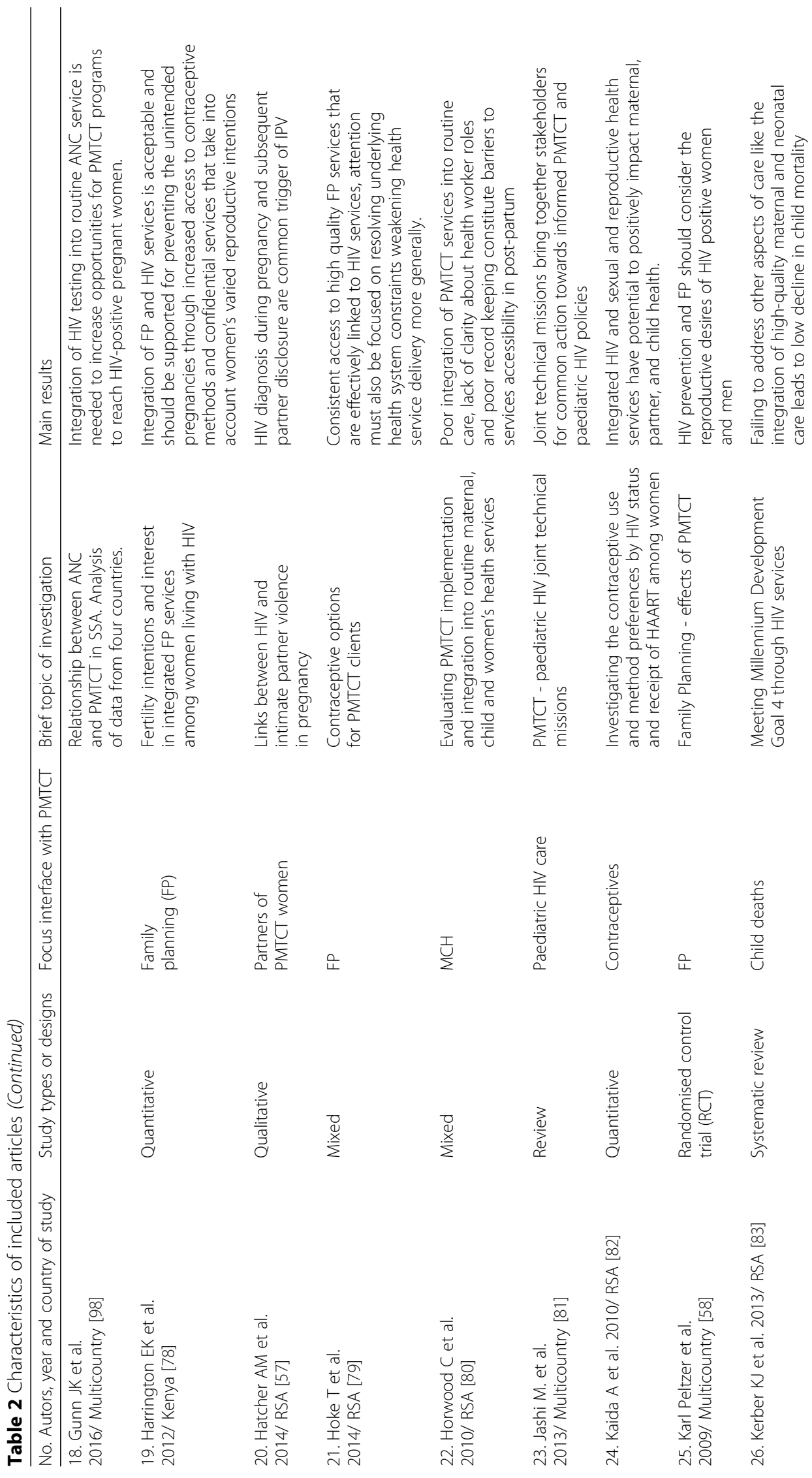




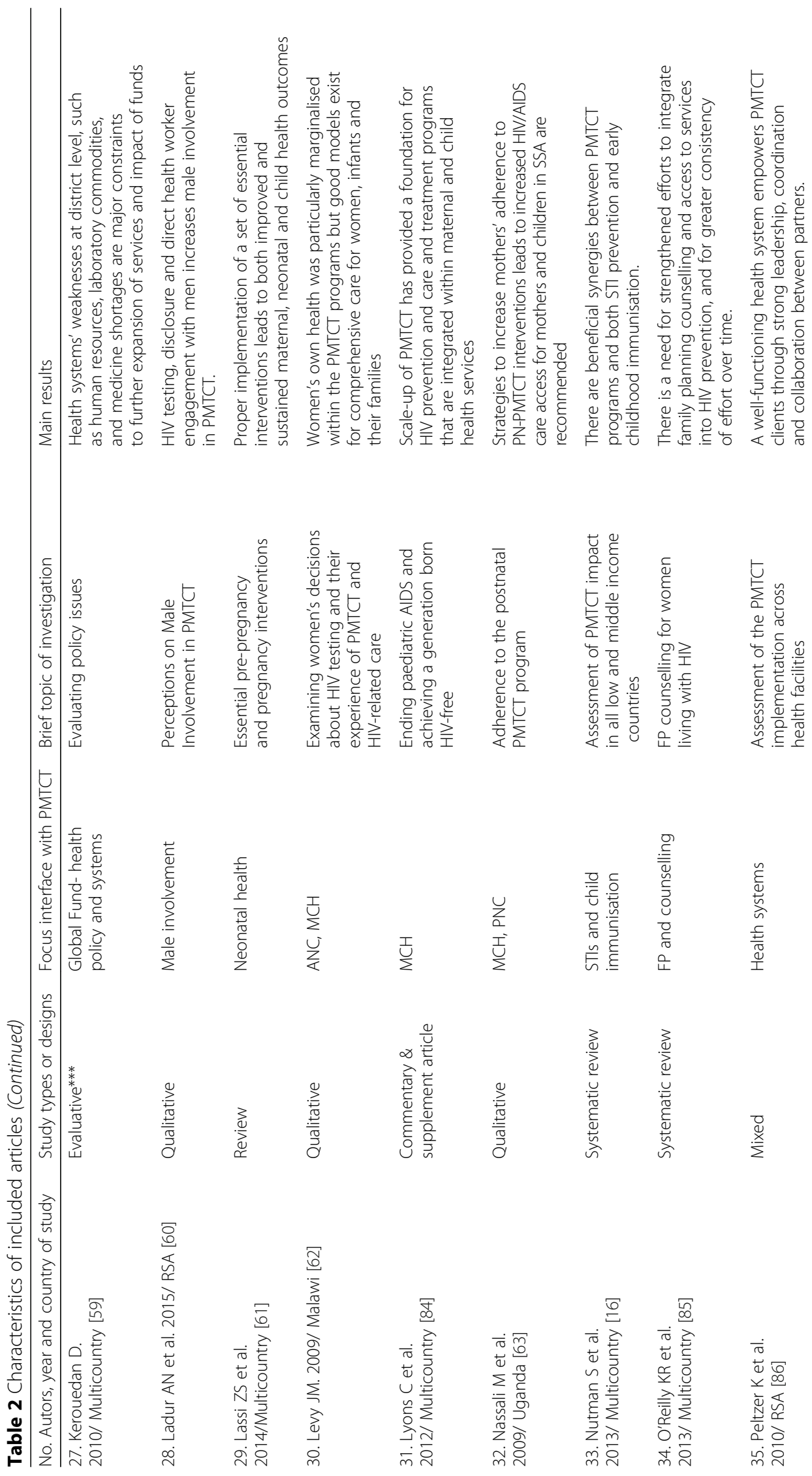




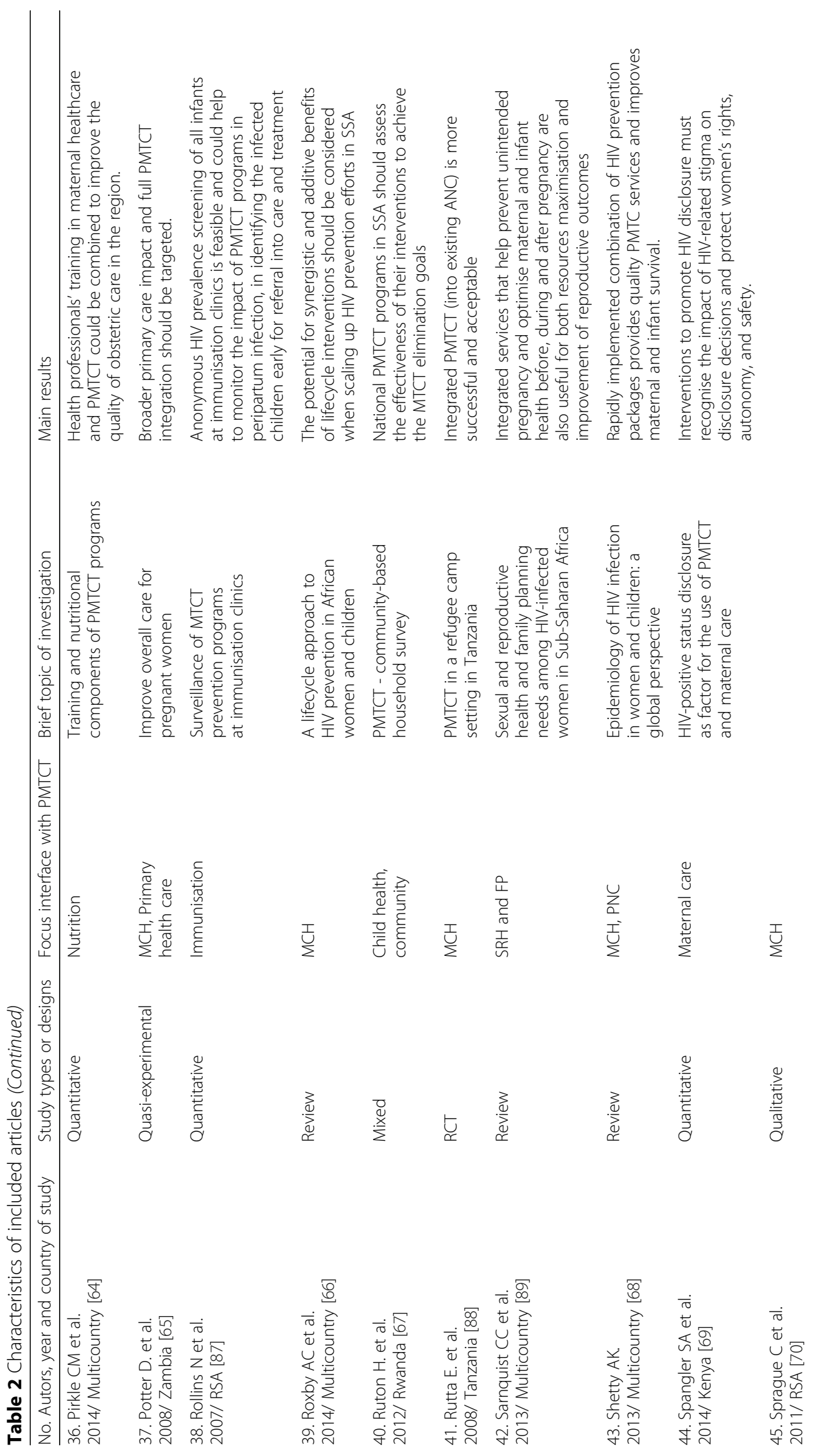




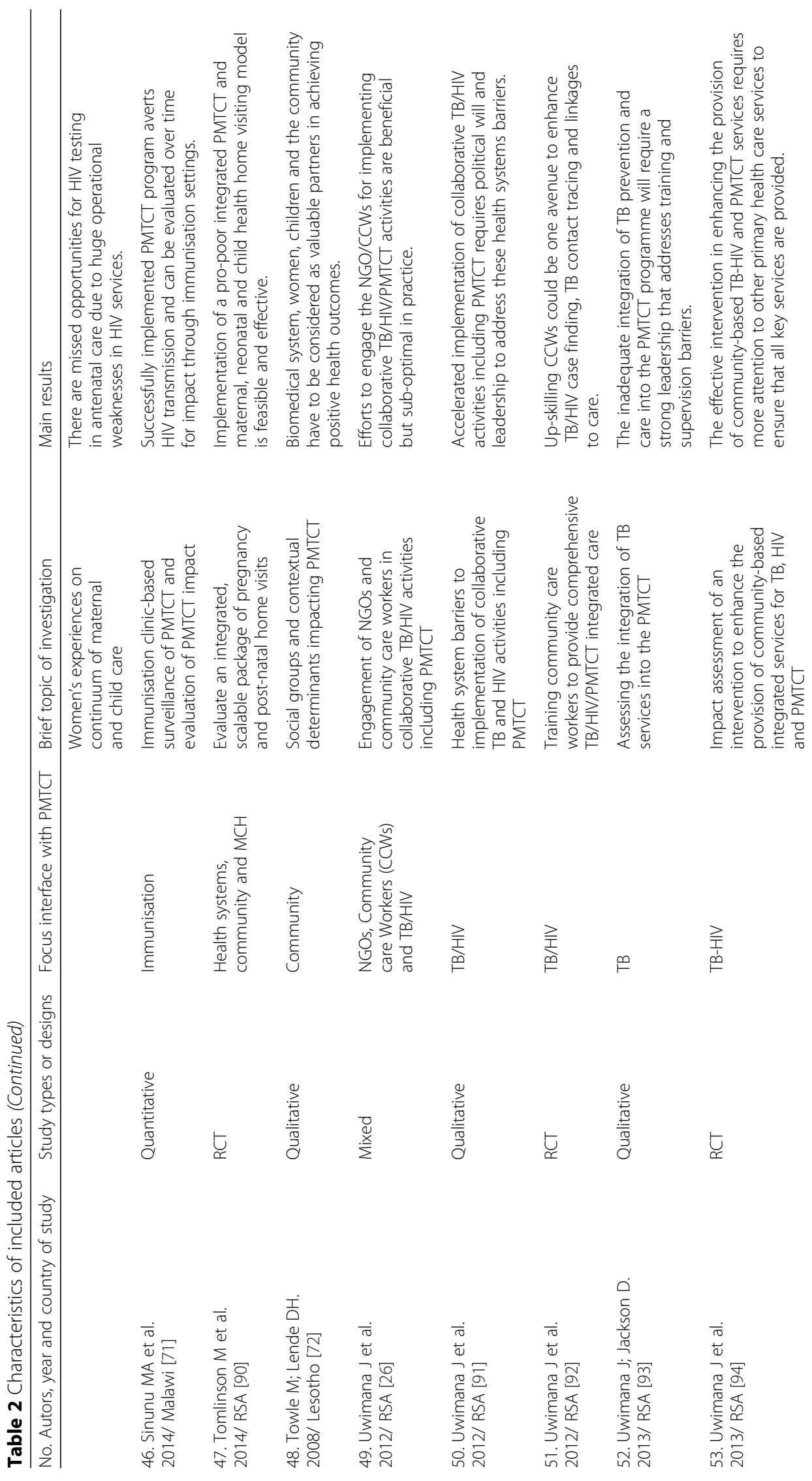




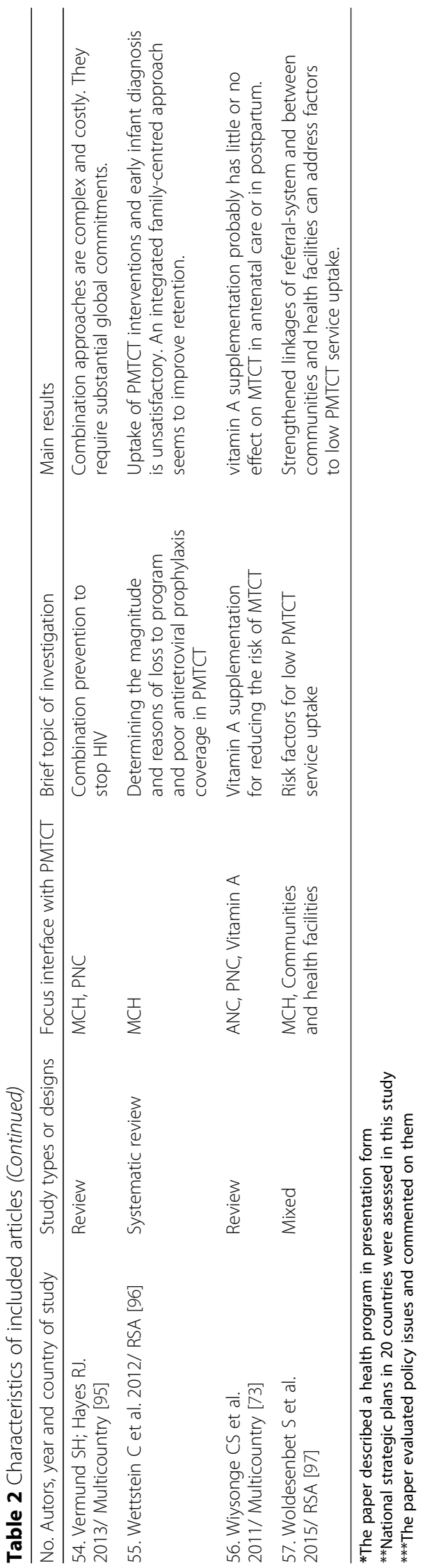


among the selected areas/sites and participants. Key informants such as policy makers, district health workers, academicians, implementing partners and persons living with HIV were also contacted in the research process in most of selected articles. Many programs and services were classified as fully vertical while a few others were perceived as either semi or fully integrated based on the extent of verticality or horizontality of PMTCT programs [14, 44].

Thirty-two articles recorded the positive and negative effects of PMTCT on other health care services as a major theme in the article's title and/or discussion, out of fiftyseven [14, 16, 45-74]. Twenty-five articles examined PMTCT and its integration in health systems in SSA as a main theme [26, 44, 75-97]. Twenty-three papers out of 57 were classified as multinational as they covered two or more SSA countries $[16,44,47,52,54-56,59,61,64,66,68,73,74,77,81,84,85,89,95,98]$, twenty examined our research themes in the Republic of South Africa (RSA) [26, 46, 48, $51,57,60,70,79,80,82,83,86,87,90-94,96,97]$, three in Kenya [53, 69, 78], two each for Malawi $[62,71]$ and Tanzania $[76,88]$ and one for each of Democratic Republic of Congo [49], Lesotho [72], Rwanda [67], Senegal [75], Swaziland [45], Uganda [63] and Zambia [65].

Integration as a theme was found in twelve papers on PMTCT integration in ANC, postnatal care (PNC) and child care $[44,76,77,80,83,86,88,90,95-98]$, five papers examining PMTCT and HIV services integration with tuberculosis screening and treatment [26, 91-94], six articles on integrating sexual and reproductive health (SRH) and family planning (FP) services to prevent unintended pregnancy and optimise maternal and infant health in ANC and PNC [75, 78, 79, 82, 85, 89], one article on linking immunisation to HIV screening among children [87] and one on paediatric HIV [84]. We found literature examining both impact and integration of PMTCT programs as part of maternal and child health $(\mathrm{MCH})$ services. $\mathrm{MCH}$ in this review covers antenatal, delivery and post-partum or post-natal care for both the mother and child. Other elements related to direct $\mathrm{MCH}$ services were included, notably social factors such as women's perceptions, community and male involvements in mother and child services, intimate partner violence and gender equality. The above-mentioned $\mathrm{MCH}$ components appeared in a total of twenty-one articles $[14,45,47-49,53-55,57,59,60,62,63,65-70,72,74]$.

\section{Impact of PMTCT on other health care services}

PMTCT has been promoted by WHO as a reliable solution to paediatric HIV. As a comprehensive approach developed based on the four components, the scaling up of PMTCT became a cornerstone of countries' HIV prevention, care and treatment programs [42]. As mentioned above, 32 of the 57 retained articles addressed the impact of PMTCT on other health care services. The major findings are the potential improvement of existing $\mathrm{MCH}$ services and to some extent increasing the availability, accessibility and utilisation of other PMTCT-related services such as prevention of unintended pregnancies, control of STI, immunisation, nutrition and vitamins supplementation $[16,44,73,76,78,87,89]$. PMTCT services also offer, to some extent, opportunities for screening tuberculosis among exposed infants [51] and may generally enhance diagnosis and treatment through collaboration efforts at both health care settings and community [26, 91, 92, 94]. 
Several issues surrounding the PMTCT roll-out are reported to adversely impact PMTCT outcomes [14] and these include its process, services delivery, quality of supplies and tools, hiring and training of adequate staff, among others. However, conclusions regarding the impact of PMTCT must be cautious due to lack of detailed studies analysing such effects. In their attempt to quantify the impact of PMTCT programs on overall health systems in SSA, Nutman et al. [16] specifically assessed the existing knowledge and evaluated the PMTCT services impact beyond HIV transmission prevention. They also looked at how these programs contribute to the broader health outcomes. In the end, their systematic review of literature published up to 2011 found evidence of numerous beneficial synergies with specific health services but that there was insufficient evidence to draw any firm conclusions about the broader impacts of PMTCT on health outcomes or health systems. They report serious gaps regarding appropriate recording, data availability and information flow, and argue that these gaps might mislead decision-making, funding allocation and implementation of initiatives.

\section{Health system strengthening or weakening}

Health systems link people with promotive, preventive, curative, rehabilitative or palliative health services to address health problems. The effectiveness of this linkage depends on many factors, including those outside the health sector as well as within the various components of a health system [99, 100]. We identified six articles explicitly or indirectly addressing the broader health system $[44,46,59,86,90,91]$ while the remaining fifty-one focused internally, on one or more components of the building blocks and functioning of health systems and services.

Health care providers and funders across SSA have shown interest and engagement to make PMTCT services more and easily accessible to women and children [16, 47, 84, 101] but their efforts have not eliminated many challenges underlying SSA health systems. Added to social problems, these health system issues create a situation in which it is difficult for women to actually utilise services during pregnancy and postpartum, even when these are offered free of charge [76, 102, 103]. Looking within the health systems themselves, PMTCT services have positively impacted $\mathrm{MCH}$ and adequately reduced the spread of HIV infection [16], but optimal outcomes occur where the health services are delivered in conducive working conditions, with adequately equipped facilities and committed management [104]. Such conditions are rare in SSA where the vertical transmission of HIV is the highest in the world. PMTCT and other HIV services in SSA depend on foreign funds estimated at billions of dollars but unfortunately the overall system and population-level results of such efforts and investments are seen as mixed [21].

Studies in this review pointed to these shortcomings and deficiencies in service delivery, especially for the components regarding the uptake of PMTCT programs such as antenatal HIV testing and receipt of test results, ARV prophylaxis and postnatal mother-infant follow up $[105,106]$. Campbell et al. [21] therefore argue for a strategy for (i) 'translating' intervention approaches into locally and culturally appropriate discourses and practices; (ii) building local capacity to sustain interventions once their funded period is over; and (iii) strengthening health systems in affected settings. 
Analysing the effects of PMTCT on other health care services is often framed in terms of a debate over whether the roll-out of HIV/AIDS services including PTMTC did or did not strengthen the existing health systems and services. Studies in our review demonstrated the advantages of scaling up HIV services in terms of saving many lives, training health providers, and funding some key services like $\mathrm{MCH}$, but the same findings highlighted the various shortcomings of HIV/AIDS interventions in health systems of low and middle-income countries and particularly in SSA [14, 27, 44, 65, 102, 106]. Some encouraging results suggest positive impact of PMTCT programs on health systems [44] but also highlight the challenges that PMTCT and health systems did not meet; these unmet challenges are seen as negative effects mostly caused by poorly resourced settings facing a high disease burden [14]. These challenges include among others: continued high rates of home deliveries, shortages of personnel, inadequate supplies of test kits, varying distribution and availability of PMTCT service delivery points, lack of supplementary feeds for women who may opt for non-breast feeding for their infants, and logistical and social implications after testing HIV positive, such as a lack of spousal support and sometimes violence $[14,103,106,107]$. None of the retained papers showed or argued that PMTCT programs directly weaken health systems. In order to strengthen health systems, the implementation of PMTCT and disease-specific interventions needs more collaborative efforts to address structural, organisational, managerial and finance barriers.

\section{Integration of PMTCT within broader programs and health systems}

Even though some programs remain disease-specific, our review found that PMTCT programs and other health services mutually interact. PMTCT integration within general health care services in low and middle income countries has also been recommended to boost the utilisation of these interventions [27]. Some studies noted that under a PMTCT umbrella, the quality of other services in integration is also being closely monitored and improved [102, 105].

PMTCT integration has for example, positively influenced maternal and child care services regarding service availability, accessibility and utilisation [44]. Evjen-Olsen et al. [76] suggested that maternal and neonatal health can be improved by integrating health care services, supporting integration of health systems rather than separately organising and managing different vertical and horizontal programs especially in developing countries [108]. The articles reviewed here also identified potential synergies between the integration or combination of PMTCT with specific health care activities outside of direct obstetrical and child care or $\mathrm{MCH}$ services, including sexually transmitted infection (STI) control and immunisation [16, 71], sexual and reproductive health and family planning [56, 58, 61], nutrition [64], tuberculosis [51] and vitamin A supplementation [73]. The synergies are variously achieved in different contexts through progressive efforts, such as staff training and motivation, planning and evaluation of services, restructured management and financing among others. In terms of integration itself as a theme, the following synergies were examined by retained studies, apart from MCH: HIV/PMTCT services integration with tuberculosis screening and treatment [26, 91-94], with SRH, STIs and FP services [16, 75, 78, 79, 82, 85, 89] and with immunisation and HIV screening among children [87]. These linkages helped to increase and improve training of care providers, to review and enhance funding and 
implementation policies, to increase access and adherence to services, to reduce drug stock outs and improve basic infrastructures.

While identifying and describing the effects of PMTCT on other health services and health systems or the integration of PMTCT and its extent within broader programs and health systems, collaborations and involvements at different levels emerged to be crucial. Since health services are not only provided by the public sector, our search included other organisations offering PMTCT services and other actors involved in offering such services outside of the public sector. We identified examples of service delivery provided by NGOs [26], or delivered in refugee camps [88] while one paper focused on male partner involvement [60]. While studies examining public health services tended to focus on activities within clinics and services, these additional papers reveal that PMTCT activities shape interactions between the community members, social organisations and clinics offering the PMTCT services. The collaborations reported in some articles were directed to implementation or evaluation of initiatives against stigma, for the "normalisation" of HIV as just another other disease and to increase the accessibility of other social and support services to women living with HIV [6]. Selected articles stressed collaboration initiatives so that the PMTCT programs could become a model in "networking, nurturing relationships and bringing all available resources and agents to the table to find solutions and forge partnerships in order to procure all elements essential to a high quality, comprehensive, integrated program" [42].

\section{Discussion}

The review aimed to document the possible effects of PMTCT on health care services and systems and the integration of PMTCT within broader programs and health systems in the available literature in SSA. The analysis of available evidence regarding the two main aspects of this review, namely PMTCT impact and integration, supports a generally positive evaluation of positive synergies with $\mathrm{MCH}$ as well as other health services, suggests increasing partial integration of PMTCT within health systems, and offers inconclusive arguments on whether health systems as a whole have been strengthened or weakened by the PMTCT programs in countries of SSA. The discussion below addresses three issues that must be further studied and analysed in order to increase the likelihood that health systems will be demonstrably strengthened: the availability and quality of information, synergies and impacts within and beyond the health sector and the need to engage programmes and services beyond $\mathrm{MCH}$, sexual and reproductive health, and $\mathrm{TB}$.

Beyond the structural limitations on the positive impact that PMTCT programs might have on health systems in SSA, there is also a crucial continuing problem of accurate and timely information as highlighted by Theuring et al., (22), and a lack of rigorous research regarding the effects of PMTCT programs on health care systems or vice versa. While all included papers contributed to one or another dimension of the research questions, only two among 32 retained articles that recorded the impact of PMTCT, explicitly and directly focused on or addressed the impact of PMTCT on other health services and on health systems $[14,16]$. This is a very unusual scenario, considering the large body of available literature on HIV/AIDS interventions in SSA. It is worth repeating that Nutman et al. [16] specifically aimed to evaluate the impact of 
PMTCT services beyond transmission prevention and assessed the existing knowledge about such programs and how they contribute to broader health outcomes. Unfortunately, these research efforts were stymied by the weakness of health information systems, unreliable administrative and research data, and important evidence gaps. This research gap, coupled with under- or improper reporting practices, poses serious challenges to informed decision making, proper funding allocation and effective implementation.

Likewise, in their 2011 Cochrane review, Lorainne Tudor Car and colleagues did not find any study that evaluated the PMTCT interventions integration with other health care services to improve health outcomes [27]. In another study conducted in Swaziland, a country with a background of high, but stable HIV prevalence, an intervention with triangulated data of HIV testing and counselling, ART, PMTCT, and TB screening programs improved coverage and documented promising health outcomes. Even though the scale-up of this intervention was successful, the lack of data remained a non-negligible challenge throughout the study [109]. A situation like this is not an exception in SSA where health records are often incomplete or poorly managed. Without data storage and sharing, the combination of health interventions becomes more complicated and even impossible. This tendency can only be changed at national and regional levels through viable, coordinated investment and practice to monitor and to improve effects of major programs like PMTCT on other services and health systems or vice versa.

\section{Synergies, collaborations and impacts on health systems: Positive contributions to a problem beyond the scope of PMTCT and the health sector}

The included papers provided moderate evidence of mutual benefits between the PMTCT services and the existing health care services and they recommended a close relationship and more integration in order to maximise the advantages of working together and to mitigate some of the challenges of controlling HIV, a lifelong condition, in the conditions facing SSA $[8,14,27]$. The available scientific studies and reports suggest the existence of both positive and negative effects generated by PMTCT on the maternal health services where they are mostly based and on child health services. The PMTCT services increase not only the accessibility but also the utilisation of antenatal and other $\mathrm{MCH}$ services [110]. In addition, the increased access to HIV testing and treatment are transforming HIV into a chronic illness. However, the lifelong management of HIV requires continued commitment and engagement of different actors such as the funders, the health care system, the patients, their families and as well as the community [4]. The efforts to manage the HIV pandemic as a chronic disease and treat the opportunistic infections or any other HIV associated conditions overwhelm SSA's struggling health care systems. The systems that depend on conditional foreign aid do not enjoy managerial autonomy and cannot consistently plan for their future interventions. PMTCT services within such systems cannot, on their own, strengthen health systems.

As seen in the results section, the failure of PMTCT to demonstrably strengthen health systems is associated and sustained by the underfunded and poorly managed health care settings [14]. HIV counselling and testing is not yet universal and many pregnant women come late to seek prenatal care and they then access all the services 
at the same time instead of progressing through the whole cascade. Lack of adequate resources and facilities (infrastructure, materials, and human resources among others) are major barriers to successful interventions in SSA where the operational and implementation problems arise from local contextual issues as well as the underlying poverty reflecting global economic forces.

The reality of plural health systems and multiple actors must also be addressed in research, policy, and practice. In SSA, the private for-profit sector and charitable and faith-based health care providers including mission hospitals and NGOs play an important role $[26,91]$ and should be included in research and policy dialogue. This entails the widely advocated but in practice difficult coordination between well-funded international organisations, struggling national public health systems, NGOs and people living with HIV in search of treatment [111].

Beyond general statements of goals of collaboration and consideration of intervention context, there should be a clear strategy to strengthen local responses to HIV which are often ignored by the top-down style adopted in order to comply with requirements of "the global funding architecture" [112]. This responsiveness to local conditions and actors takes time and requires flexibility. In contrast, the "emergency" nature of much HIV intervention combined with accountability requirements to funders tend to align with a more directive and hence less responsive and collaborative approach. Constructive collaboration is not only advised but critically needed in order to achieve both PMTCT and health services goals. This concerns all models of health care delivery, whether vertical, horizontal or diagonal.

\section{Beyond $\mathrm{MCH}$, sexual and reproductive health and TB}

Malaria, severe anaemia, diarrheal diseases and acute respiratory diseases are some of the leading causes of death among women and children in many of SSA countries $[14,113]$ including RSA, the source of most studies identified for this review. These are rarely, if at all addressed in PMTCT programs and their impacts on PMTCT or vice versa are not discussed in any study included in this review. This is possibly due to HIV interventions and research continuing to be seen as a separate issue from health conditions other than those directly related to sexual and reproductive health and TB.

In the cited systematic review of Lorainne Tudor Car et al. [27] on integrating PMTCT programs with other health services for preventing HIV infection and improving HIV outcomes in developing countries, the authors decided not to make any recommendation about the implementation of integrated PMTCT programs based on the fact that only one study met their selection criteria. It is logical to conceive and integrate the PMTCT programs within MCH services given the correlation of both services delivery but other services such as SRH or immunisation clinics as the point of entry to PMTCT programs need to be explored. In addition to publications for this review that recommend more research on the impact of PMTCT programs on health care services, the WHO, UNAIDS, UNICEF, UNFPA and PEPFAR calls to integrate the PMTCT and other disease stand-alone programs with other health care services $[6,10,12,42]$ remain relevant.

\section{Limitations}

This a comprehensive and complex review that included a range of issues related to PMTCT impact and its integration on other health services and systems. Even though 
it was rigorously conducted, not all PMTCT implementation details could be reviewed in this single paper.

The regional HIV prevalence and other crucial factors, such as relationships with donors and international researchers as well as publication outputs, are highly variable across countries and regions in SSA. Most of the identified studies covered eastern and southern Africa. Central and western parts of Africa are less covered in publications, a situation that makes it difficult to generalise this review's findings to those regions. Studies reporting good service delivery and adherence may also not reflect the full impact of PMTCT when implemented at scale at a national level. Some areas within countries may also have been left out in the retrieved studies.

As this review sought articles specifically addressing PMTCT and health services and systems, it may have missed publications reflecting recent increased attention and funding for health system strengthening through HIV programmes, which did not specifically address PMTCT. These papers may yield insights into broader health system challenges and promising approaches to integration of other HIV programmes, potentially including PMTCT.

\section{Conclusion}

PMTCT improves maternal and child health through preventing the spread of HIV infection in SSA countries. There is evidence of a positive impact of PMTCT on primary care for mothers and children, beyond HIV. The provision of PMTCT services increases the availability, the accessibility and the utilisation of antenatal care and other health care services, especially when the intervention is linked to PMTCT programs as part of mainstream $\mathrm{MCH}$ services. However, this review also documented a large number of challenges involved both in implementing and in understanding the effects of PMTCT integration. Without robust information systems and rigorous and systematic research on the health system as a whole as well as on its various services and activities, evidence will continue to be fragmented and firm conclusions will continue to be impossible to draw.

While some vertical programs persist, PMTCT services are increasingly being integrated at different levels within routine health services and the health systems. Our study documented the challenges and weaknesses that face health care services and health systems in connection with PMTCT services. These range from structural, governance and resourcing challenges within and between countries, to weak information reporting systems and require more and better coordination and collaboration within and beyond HIV programs, directly related health services, communities, the plural health sector, and other sectors at national and global levels. These broad health system and social challenges cannot be solved by PMTCT interventions alone and there is a need of working together or collaborating with other sectors out of health system.

\section{Abbreviations}

AIDS: Acquired Immune Deficiency Syndrome; ANC: Antenatal care; ART: Antiretroviral therapy; ARV: Antiretrovirals; CCWs: Community care workers; FP: Family planning; HIV: Human Immunodeficiency Virus; MCH: Maternal and child health; MTCT: The Mother-To-Child Transmission of HIV; NGOs: Non-Governmental Organisations; PMTCT: Prevention of mother-to-child transmission of HIV; PNC: Postnatal care; RSA: The Republic of South Africa; SRH: Sexual and reproductive health; SSA: Sub-Saharan Africa; STI: Sexually Transmitted Infection; TB: Tuberculosis; UNAIDS: The United Nations Programme on HIV and AIDS; UNFPA: The United Nations Population Fund; UNGASS: The United Nations General Assembly Special Session; UNICEF: The United Nations Children's Fund; WHO: World Health Organisation 


\section{Acknowledgements}

We would like to thank Sylvie Fontaine, the librarian at paramedical library, Université de Montréal, Canada, for her valuable contribution to search strategy development.

\section{Funding}

No funding has been received for the review protocol or the review. MJC holds a PhD scholarship of her supervisor Christina Zarowsky from the Canadian Institutes of Health Research (CIHR) under the "Team Grant - Implementation Research in the Prevention and Treatment of Type II Diabetes in Low and Middle Income Countries" competition. This funding is for the following randomised trial: integrated health system intervention aimed at reducing type 2 diabetes risk in women after gestational diabetes in South Africa (IINDIAGO). HT holds a salary award (chercheur-boursier) from the "Fond de la recherche en santé- du Québec (FRQ-S) and a salary award (New Investigator Salary Award) from Canadian Institutes of Health Research (CIHR)".

\section{Availability of data and materials}

Not applicable.

\section{Authors' contributions}

MJC, CZ, and HT designed the study. MJC wrote the first manuscript of the review. CZ and HT critically revised the review. All authors read and approved the final manuscript.

\section{Authors' information}

MJC is a MHA, MSC, MA, MPhil and PhD candidate in Global Health. CZ is a MD, MPH and PhD in Medical Anthropology. HT is a M.Sc and PhD in Epidemiology.

\section{Ethics approval and consent to participate}

Not applicable.

\section{Consent for publication}

Not applicable.

\section{Competing interests}

The authors declare that they have no competing interests.

\section{Publisher's Note}

Springer Nature remains neutral with regard to jurisdictional claims in published maps and institutional affiliations.

\section{Author details}

'Département de Médecine Sociale et Préventive, École de Santé Publique, Université de Montréal, Pavillon 7101 , Avenue du Parc, Montreal, QC H3N 1X7, Canada. ${ }^{2}$ Centre de Recherche du Centre Hospitalier de I'Université de Montréal (CRCHUM), Tour Saint-Antoine, 3rd Floor, Room: S03.516, 900, Rue St-Denis, Montreal, QC H2X 0A9, Canada. ${ }^{3}$ Centre de Recherche du Centre Hospitalier de I'Universitaire Sainte Justine, Montréal, QC H3T 1C5, Canada. ${ }^{4}$ School of Public Health, University of the Western Cape, Robert Sobukwe Rd, Bellville 7535, South Africa.

Received: 27 June 2017 Accepted: 9 October 2017

Published online: 05 December 2017

\section{References}

1. UNAIDS. Global report: UNAIDS report on the global AIDS epidemic 2013. Geneva: UNAIDS; 2013. p. 198.

2. UNAIDS. HIV/AIDS fact sheet. 2016 [cited 2017

3. Mahy $\mathrm{M}$, et al. What will it take to achieve virtual elimination of mother-to-child transmission of HIV? An assessment of current progress and future needs. Sex Transm Infect. 2010;86(Suppl 2):ii48-55.

4. Psaros C, et al. Adherence to HIV care after pregnancy among women in sub-Saharan Africa: falling off the cliff of the treatment cascade. Curr HIV/AIDS Rep. 2015;12(1):1-5.

5. AVERT. Prevention of mother-to-child transmission (PMTCT) of HIV. 2016 30.01.2017]; Available from: http://www. avert.org/professionals/hiv-programming/prevention/prevention-mother-child.

6. WHO. PMTCT strategic vision 2010-2015: preventing mother-to-child transmission of HIV to reach the UNGASS and millennium development goals: moving towards the elimination of paediatric HIV. 2010 [cited 2016 23/09/ 2016]; Available from: http://www.who.int/hiv/pub/mtct/strategic_vision.pdf.

7. UNAIDS. UNAIDS calls for a virtual elimination of mother to child transmission of HIV by 2015. Geneva: UNAIDS 2009 .

8. Rollins N, et al. Prioritizing the PMTCT implementation research agenda in 3 African countries: INtegrating and Scaling up PMTCT through Implementation REsearch (INSPIRE). J Acquir Immune Defic Syndr. 2014;67(Suppl 2): S108-13.

9. WHO. Consolidated guidelines on HIV prevention, diagnosis, treatment and care for key populations. 2016 [cited 2016 23/09/2016]; Available from: http://www.who.int/hiv/pub/guidelines/keypopulations-2016/en/.

10. WHO \& UNICEF. Guidance on global scale-up of the prevention of mother-to-child transmission of HIV: towards universal access for women, infants and young children and eliminating HIV and AIDS among children. 2007 [cited 2016 01/06/2016]; Available from: https://www.unicef.org/aids/files/PMTCT_enWEBNov26.pdf.

11. Brou $\mathrm{H}$, et al. When do HIV-infected women disclose their HIV status to their male partner and why? A study in a PMTCT programme, Abidjan. PLoS Med. 2007;4(12):e342. 
12. WHO \& UNICEF. Global monitoring framework and strategy for the global plan towards the elimination of new HIV infections among children by 2015 and keeping their mothers alive (EMTCT). 2012; Available from: http://www.who.int/hiv/pub/me/monitoring_framework/en/.

13. Schechter J, et al. Exploring loss to follow-up among women living with HIV in prevention of mother to child transmission programmes in cote d'Ivoire. Glob Public Health. 2014;9(10):1139-51.

14. Both JM, van Roosmalen J. The impact of Prevention of Mother to Child Transmission (PMTCT) programmes on maternal health care in resource-poor settings: looking beyond the PMTCT programme-a systematic review. BJOG. 2010;117(12):1444-50.

15. Ooms G, et al. The 'diagonal' approach to Global Fund financing: a cure for the broader malaise of health systems? Glob Health. 2008;4(1):6.

16. Nutman S, McKee D, Khoshnood K. Externalities of prevention of mother-to-child transmission programs: a systematic review. AIDS Behavior. 2013;17(2):445-60.

17. UNAIDS, Global report. UNAIDS report on the global AIDS epidemic 2013. 2013.

18. WHO. World AIDS day celebration in Rwanda. 2014 23/09/2016]; Available from: http:/www.afro.who.int/en/ rwanda/press-materials/item/7280-world-aids-day-celebration-in-rwanda.html.

19. WHO. WHO regional director for Africa commends South Africa for its leadership in HIV, TB and PMTCT prevention and control. 2014 [cited 23/09/2016; Available from: http://www.afro.who.int/en/south-africa/pressmaterials/item/6444-who-regional-director-for-africa-commends-south-africa-for-its-leadership-in-hiv-tb-and-pmtctprevention-and-control.html.

20. Nguyen, V.-K., Antiretroviral globalism, biopolitics, and therapeutic citizenship. 2005.

21. Campbell C, Cornish F, Skovdal M. Local pain, global prescriptions? Using scale to analyse the globalisation of the HIV/AIDS response. Health Place. 2012;18(3):447-52.

22. Nguyen VK, et al. Adherence as therapeutic citizenship: impact of the history of access to antiretroviral drugs on adherence to treatment. AIDS. 2007;21(Suppl 5):S31-5.

23. Theuring S, et al. Male involvement in PMTCT services in Mbeya Region, Tanzania. AIDS Behav. 2009;13(1):92-102.

24. Binagwaho A, et al. Scaling up early infant diagnosis of HIV in Rwanda, 2008-2010. J Public Health Policy. 2013;34(1):2-16.

25. Wilmoth J, et al. Maternal deaths drop by one-third from 1990 to 2008: a United Nations analysis. Bull World Health Organ. 2010;88(10):718-718a.

26. Uwimana J, et al. Engagement of non-government organisations and community care workers in collaborative TB/HIV activities including prevention of mother to child transmission in South Africa: opportunities and challenges. BMC Health Serv Res. 2012;12:233.

27. Tudor Car $L$, et al. Integrating prevention of mother-to-child HIV transmission (PMTCT) programmes with other health services for preventing HIV infection and improving HIV outcomes in developing countries. Cochrane Database Syst Rev. 2011;(6):Cd008741.

28. Oliveira-Cruz V, Kurowski C, Mills A. Delivery of priority health services: searching for synergies within the vertical versus horizontal debate. J Int Dev. 2003;15(1):67-86.

29. Frenk J. The global health system: strengthening national health systems as the next step for global progress. PLoS Med. 2010;7(1):e1000089.

30. Shigayeva A, et al. Health systems, communicable diseases and integration. Health Policy Plan. 2010;25(Suppl 1):i4-20.

31. Tudor Car L, et al. Integrating prevention of mother-to-child HIV transmission programs to improve uptake: a systematic review. PLoS One. 2012;7(4):e35268.

32. Atun $\mathrm{R}$, et al. A systematic review of the evidence on integration of targeted health interventions into health systems. Health Policy Plan. 2010;25(1):1-14.

33. Mazia G, et al. Integrating quality postnatal care into PMTCT in Swaziland. Glob Public Health. 2009:4(3):253-70.

34. Maher D, Harries A, Getahun H. Tuberculosis and HIV interaction in sub-Saharan Africa: impact on patients and programmes; implications for policies. Tropical Med Int Health. 2005;10(8):734-42.

35. van Eijk AM, et al. Malaria and human immunodeficiency virus infection as risk factors for anemia in infants in Kisumu, western Kenya. Am J Trop Med Hyg. 2002;67(1):44-53.

36. ter Kuile FO, et al. The burden of co-infection with human immunodeficiency virus type 1 and malaria in pregnant women in sub-saharan Africa. Am J Trop Med Hyg. 2004;71(2 Suppl):41-54.

37. Oliveira C, Russo G. Vertical interventions and system effects; have we learned anything from past experiences? Pan African Medical Journal. 2015;21:262.

38. Taylor CE, Waldman R. Designing eradication programs to strengthen primary health care, in The eradication of infectious disease: report of the Dahlem Workshop on the Eradication of Infectious Diseases. Chichester: Wiley; 1998. p. 145-55.

39. Grant MJ, Booth A. A typology of reviews: an analysis of 14 review types and associated methodologies. Health Inf Libr J. 2009;26(2):91-108.

40. Reuters T. EndNote X2. Carlsbad: Thomson Reuters; 2008.

41. Frieden TR. A framework for public health action: the health impact pyramid. Am J Public Health. 2010;100(4):590-5.

42. PEPFAR, PEPFAR guidance on integrating prevention of mother to child transmission of HIV, maternal, neonatal, and child health and pediatric HIV services. 2011.

43. Nakakeeto ON, Umaranayake L. The global strategy to eliminate HIV infection in infants and young children: a seven-country assessment of costs and feasibility. AIDS. 2009;23(8):987-95.

44. Druce N, Nolan A. Seizing the big missed opportunity: linking HIV and maternity care services in sub-Saharan Africa. Reproductive Health Matters. 2007;15(30):190-201.

45. Bancheno WM, Mwanyumba F, Mareverwa J. Outcomes and challenges of scaling up comprehensive PMTCT services in rural Swaziland, Southern Africa. AIDS Care. 2010;22(9):1130-5.

46. Barker PM, Mphatswe W, Rollins N. Antiretroviral drugs in the cupboard are not enough: the impact of health systems' performance on mother-to-child transmission of HIV. Journal of Acquired Immune Deficiency Syndromes: JAIDS. 2011;56(2):e45-8.

47. Barker PM, Mate K. Eliminating mother-to-child HIV transmission will require major improvements in maternal and child health services. Health Aff. 2012;31(7):1489-97. 
48. Barry OM, et al. Development of a measure of the patient-provider relationship in antenatal care and its importance in PMTCT. AIDS Care. 2012;24(6):680-6.

49. Behets F, et al. Reducing vertical HIV transmission in Kinshasa, Democratic Republic of Congo: trends in HIV prevalence and service delivery. AIDS Care. 2009;21(5):583-90.

50. Chi BH, Stringer JS, Moodley D. Antiretroviral drug regimens to prevent mother-to-child transmission of HIV: a review of scientific, program, and policy advances for sub-Saharan Africa. Current HIV/AIDS Reports. 2013;10(2):124-33.

51. Cotton MF, et al. Tuberculosis exposure in HIV-exposed infants in a high-prevalence setting. International Journal of Tuberculosis \& Lung Disease. 2008;12(2):225-7.

52. DeGennaro V, Zeitz P. Embracing a family-centred response to the HIV/AIDS epidemic for the elimination of pediatric AIDS. Global Public Health. 2009;4(4):386-401.

53. du Plessis E, et al. Prevention of mother-to-child transmission of HIV in Kenya: challenges to implementation. BMC Health Serv Res. 2014;14(Suppl 1):S10.

54. Ekouevi DK, et al. Health facility characteristics and their relationship to coverage of PMTCT of HIV services across four African countries: the PEARL study. PLoS One. 2012;7(1):e29823.

55. Gibbs A, et al. The inclusion of women, girls and gender equality in National Strategic Plans for HIV and AIDS in southern and eastern Africa. Global Public Health. 2012;7(10):1120-44.

56. Govender T, Coovadia $\mathrm{H}$. Eliminating mother to child transmission of HIV-1 and keeping mothers alive: recent progress. J Infect. 2014;68(Suppl 1):S57-62.

57. Hatcher AM, et al. Bidirectional links between HIV and intimate partner violence in pregnancy: implications for prevention of mother-to-child transmission. J Int AIDS Soc. 2014;17:19233.

58. Peltzer K, Chao LW, Dana P. Family planning among HIV positive and negative prevention of mother to child transmission (PMTCT) clients in a resource poor setting in South Africa. AIDS Behav. 2009;13(5):973-9.

59. Kerouedan D. The Global Fund to fight HIV/AIDS, TB and malaria 5-y: evaluation policy issues. Bull Soc Pathol Exot. 2010;103(2):119-22.

60. Ladur AN, Colvin CJ, Stinson K. Perceptions of community members and healthcare workers on male involvement in prevention of mother-to-child transmission Services in Khayelitsha, Cape Town, South Africa. PLoS One. 2015; 10(7):e0133239.

61. Lassi ZS, et al. Essential pre-pregnancy and pregnancy interventions for improved maternal, newborn and child health. Reprod Health. 2014;11(Suppl 1):S2.

62. Levy JM. Women's expectations of treatment and care after an antenatal HIV diagnosis in Lilongwe, Malawi. Reproductive Health Matters. 2009;17(33):152-61.

63. Nassali M, et al. Access to HIV/AIDS care for mothers and children in sub-Saharan Africa: adherence to the postnatal PMTCT program. AIDS Care. 2009;21(9):1124-31.

64. Pirkle CM, et al. Training and nutritional components of PMTCT programmes associated with improved intrapartum quality of care in Mali and Senegal. Int J Qual Health Care. 2014;26(2):174-83.

65. Potter D, et al. Do targeted HIV programs improve overall care for pregnant women?: Antenatal syphilis management in Zambia before and after implementation of prevention of mother-to-child HIV transmission programs. J Acquir Immune Defic Syndr. 2008;47(1):79-85.

66. Roxby AC, et al. A lifecycle approach to HIV prevention in African women and children. Current HIV/AIDS Reports. 2014;11(2):119-27.

67. Ruton $\mathrm{H}$, et al. HIV-free survival among nine- to 24-month-old children born to HIV-positive mothers in the Rwandan national PMTCT programme: a community-based household survey. J Int AIDS Soc. 2012;15:4-4.

68. Shetty AK. Epidemiology of HIV infection in women and children: a global perspective. Curr HIV Res. 2013;11(2):81-92.

69. Spangler SA, et al. HIV-positive status disclosure and use of essential PMTCT and maternal health services in rural Kenya. Journal of Acquired Immune Deficiency Syndromes: JAIDS. 2014;67(Suppl 4):S235-42.

70. Sprague C, Chersich MF, Black V. Health system weaknesses constrain access to PMTCT and maternal HIV services in South Africa: a qualitative enquiry. AIDS Research \& Therapy. 2011;8:10.

71. Sinunu MA, et al. Evaluating the impact of prevention of mother-to-child transmission of HIV in Malawi through immunization clinic-based surveillance. PLoS One. 2014;9(6):e100741.

72. Towle M, Lende DH. Community approaches to preventing mother-to-child HIV transmission: perspectives from rural Lesotho. Afr J AIDS Res. 2008;7(2):219-28.

73. Wiysonge CS, et al. Vitamin A supplementation for reducing the risk of mother-to-child transmission of HIV infection. Cochrane Database Syst Rev. 2011;1:CD003648.

74. Chi BH, Stringer JSA, Moodley D. Antiretroviral drug regimens to prevent mother-to-child transmission of HIV: a review of scientific, program, and policy advances for sub-Saharan Africa. Current HIV/AIDS reports. 2013;10(2):124-33.

75. Desclaux A, et al. Moving from prevention to elimination of mother-to-child transmission of HIV before 2015, an unrealistic objective? Social stakes in Senegal. Medecine et Sante Tropicales. 2012;22(3):238-45.

76. Evjen-Olsen B, Olsen ØE, Kvåle G. Achieving progress in maternal and neonatal health through integrated and comprehensive healthcare services-experiences from a programme in northern Tanzania. Int J Equity Health. 2009;8(1):1.

77. Ferguson L, et al. Linking women who test HIV-positive in pregnancy-related services to long-term HIV care and treatment services: a systematic review. Tropical Med Int Health. 2012;17(5):564-80.

78. Harrington EK, et al. Fertility intentions and interest in integrated family planning services among women living with HIV in Nyanza Province, Kenya: a qualitative study. Infectious Diseases in Obstetrics \& Gynecology. 2012;2012: 809682.

79. Hoke T, et al. Expanding contraceptive options for PMTCT clients: a mixed methods implementation study in Cape Town, South Africa. Reprod Health. 2014;11(1):3.

80. Horwood C, et al. Prevention of mother to child transmission of HIV (PMTCT) programme in KwaZulu-Natal, South Africa: an evaluation of PMTCT implementation and integration into routine maternal, child and women's health services. Tropical Med Int Health. 2010;15(9):992-9.

81. Jashi $M$, et al. Informing policy and programme decisions for scaling up the PMTCT and paediatric HIV response through joint technical missions. Health Policy Planning. 2013;28(4):367-74. 
82. Kaida A, et al. Contraceptive use and method preference among women in Soweto, South Africa: the influence of expanding access to HIV care and treatment services. PLoS One. 2010;5(11):e13868.

83. Kerber KJ, et al. South African child deaths 1990-2011: have HIV services reversed the trend enough to meet millennium development goal 4? AIDS. 2013;27(16):2637-48.

84. Lyons C, et al. Ending pediatric AIDS and achieving a generation born HIV-free. J Acquir Immune Defic Syndr. 2012;60(Suppl 2):S35-8.

85. O'Reilly KR, et al. Family planning counseling for women living with HIV: a systematic review of the evidence of effectiveness on contraceptive uptake and pregnancy incidence, 1990 to 2011. BMC Public Health. 2013;13:935.

86. Peltzer K, Phaswana-Mafuya N, Ladzani R. Implementation of the national programme for prevention of motherto-child transmission of HIV: a rapid assessment in Cacadu district, South Africa. African Journal of AIDS Research. 2010;9(1):95-106

87. Rollins $N$, et al. Surveillance of mother-to-child transmission prevention programmes at immunization clinics: the case for universal screening. AIDS. 2007;21(10):1341-7.

88. Rutta E, Gongo R, Mwansasu A, Mutasingwa D, Rwegasira V, Kishumbu S, et al. Prevention of mother-to-child transmission of HIV in a refugee camp setting in Tanzania. Global Public Health. 2008;3(1):62-76.

89. Sarnquist CC, Rahangdale L, Maldonado Y. Reproductive health and family planning needs among HIV-infected women in Sub-Saharan Africa. Curr HIV Res. 2013;11(2):160-8.

90. Tomlinson M, et al. Goodstart: a cluster randomised effectiveness trial of an integrated, community-based package for maternal and newborn care, with prevention of mother-to-child transmission of HIV in a South African township. Tropical Med Int Health. 2014;19(3):256-66.

91. Uwimana J, et al. Health system barriers to implementation of collaborative TB and HIV activities including prevention of mother to child transmission in South Africa. Tropical Med Int Health. 2012;17(5):658-65.

92. Uwimana J, et al. Training community care workers to provide comprehensive TB/HIV/PMTCT integrated care in KwaZulu-Natal: lessons learnt. Tropical Med Int Health. 2012;17(4):488-96.

93. Uwimana J, Jackson D. Integration of tuberculosis and prevention of mother-to-child transmission of HIV programmes in South Africa. International Journal of Tuberculosis \& Lung Disease. 2013;17(10):1285-90.

94. Uwimana J, et al. Community-based intervention to enhance provision of integrated TB-HIV and PMTCT services in South Africa. International Journal of Tuberculosis \& Lung Disease. 2013;17(10 Suppl 1):48-55.

95. Vermund SH, Hayes RJ. Combination prevention: new hope for stopping the epidemic. Current HIV/AIDS Reports. 2013;10(2):169-86.

96. Wettstein C, et al. Missed opportunities to prevent mother-to-child-transmission: systematic review and metaanalysis. AIDS. 2012;26(18):2361-73.

97. Woldesenbet $\mathrm{S}$, et al. Missed opportunities along the prevention of mother-to-child transmission services Cascade in South Africa: uptake, determinants, and attributable risk (the SAPMTCTE). PLoS One. 2015;10(7):e0132425.

98. Gunn JK, et al. Antenatal care and uptake of HIV testing among pregnant women in sub-Saharan Africa: a crosssectional study. J Int AIDS Soc. 2016;19(1):20605.

99. Nelson EC, et al. Clinical microsystems, part 1. The building blocks of health systems. Jt Comm J Qual Patient Saf. 2008;34(7):367-78.

100. Gochman, D.S., Handbook of health behavior research II: provider determinants. 2013: Springer Science \& Business Media.

101. Baek C, Rutenberg N. Implementing programs for the prevention of mother-to-child HIV transmission in resourceconstrained settings: horizons studies, 1999-2007. Public Health Rep. 2010;125(2):293-304.

102. Bhardwaj $\mathrm{S}$, et al. Elimination of mother-to-child transmission of HIV in South Africa: rapid scale-up using quality improvement. S Afr Med J. 2014;104(3 Suppl 1):239-43.

103. Auvinen J, Kylma J, Suominen T. Male involvement and prevention of mother-to-child transmission of HIV in subSaharan Africa: an integrative review. Curr HIV Res. 2013;11(2):169-77.

104. Mkoka DA, et al. "Once the government employs you, it forgets you": health workers' and managers' perspectives on factors influencing working conditions for provision of maternal health care services in a rural district of Tanzania. Hum Resour Health. 2015;13(1):77.

105. Doherty T, et al. Improving the coverage of the PMTCT programme through a participatory quality improvement intervention in South Africa. BMC Public Health. 2009;9:406.

106. Kasenga F, et al. The implications of policy changes on the uptake of a PMTCT programme in rural Malawi: first three years of experience. Glob Health Action. 2009;2

107. Byamugisha $R$, et al. Determinants of male involvement in the prevention of mother-to-child transmission of HIV programme in Eastern Uganda: a cross-sectional survey. Reprod Health. 2010;7:12.

108. WHO. Vertical-horizontal synergy of the health workforce. 2005 [cited 2015 29.04]; Available from: http://www. who.int/bulletin/volumes/83/4/editorial10405/en/.

109. van Schalkwyk C, et al. Outcomes and impact of HIV prevention, ART and TB programs in Swaziland-early evidence from public health triangulation. PLoS One. 2013;8(7):e69437.

110. Price JE, et al. Integrating HIV clinical services into primary health care in Rwanda: a measure of quantitative effects. AIDS Care. 2009;21(5):608-14.

111. Denis P. HIV/AIDS and religion in sub-Saharan Africa: an emerging field of enquiry. Archives de sciences sociales des religions. 2013;164:43-58.

112. Kelly KJ, Birdsall K. The effects of national and international HIV/AIDS funding and governance mechanisms on the development of civil-society responses to HIV/AIDS in East and Southern Africa. AIDS Care. 2010;22(Suppl 2):1580-7.

113. Rao C, Lopez AD, Hemed Y. Disease and Mortality in Sub-Saharan Africa, in Disease and Mortality in Sub-Saharan Africa; 2006. p. 43-58. 